\title{
INFLUENCIA DO USO DE RESÍDUO DE VIDRO NAS PROPRIEDADES DE ARGAMASSAS ADESIVAS*
}

\author{
Diogo Pereira dos Santos ${ }^{1}$ \\ Afonso Rangel Garcez de Azevedo ${ }^{2}$ \\ Dylmar Penteado Dias ${ }^{3}$ \\ Jonas Alexandre 4 \\ Gustavo de Castro Xavier ${ }^{5}$ \\ Fabio Da Costa Garcia Filho ${ }^{6}$ \\ Sérgio Neves Monteiro ${ }^{7}$
}

\section{Resumo}

O uso de resíduos de vidro como aditivo mineral finamente moído, em substituição parcial de cimento e agregados é uma direção promissora para a reciclagem. Este trabalho teve como objetivo analisar a influência de resíduos do processo de corte de vidro em argamassa adesiva, como substituição parcial de cimento e agregado miúdo. O vidro em pó foi utilizado em percentuais de 10, 15 e 20\% em peso em substituição ao cimento Portland e, em outro momento, em substituição ao miúdo agregado. Verificou-se que a substituição refletiu positivamente sobre a resistência à adesão da argamassa, com o aumento da temperatura de cura.

Palavras-chave: Argamassa adesiva; Resíduo de vidro; Reciclagem de vidro; Sustentabilidade.

\section{THE INFLUENCE ON THE USE OF GLASS RESIDUE ON THE PROPERTIES OF AN ADHESIVE MORTAR}

\section{Abstract}

The use of glass waste as a finely ground mineral additive, in partial replacement of cement and aggregates is a promising direction for recycling. This work aimed to analyze the influence of residues from the glass cutting process on adhesive mortar, as partial replacement of cement and small aggregate. The powdered glass was used in percentages of 10, 15 and $20 \mathrm{wt} \%$ in substitution of Portland cement and, in another moment, in substitution of the small aggregate. It was verified that the substitution reflected positively on the adhesion resistance of the mortar, with the increase of the curing temperature.

Keywords: Adhesive mortar; Glass waste; Glass recycling; Sustentability.

\footnotetext{
Universidade Estadual do Norte Fluminense - UENF, Campos do Goytacazes, RJ, Brasil. Universidade Estadual do Norte Fluminense - UENF, Campos do Goytacazes, RJ, Brasil. Universidade Estadual do Norte Fluminense - UENF, Campos do Goytacazes, RJ, Brasil. Universidade Estadual do Norte Fluminense - UENF, Campos do Goytacazes, RJ, Brasil. Universidade Estadual do Norte Fluminense - UENF, Campos do Goytacazes, RJ, Brasil. Instituto Militar de Engenharia - IME, Rio de Janeiro, RJ, Brasil.

Instituto Militar de Engenharia - IME, Rio de Janeiro, RJ, Brasil..
} 


\section{INTRODUÇÃO}

O grande desenvolvimento tecnológico, que atualmente está sendo experimentado, aumentou significativamente a produção de bens industrializados. Além do volume de recursos minerais explorados, esse aumento também resultou em uma grande quantidade de resíduos produzidos. Por esse motivo, estudos que apontam para uma solução para este problema, seja reutilização, reciclagem, processamento ou eliminação adequada desses resíduos, são necessários. Nesse cenário, a reutilização de resíduos é apresentada como uma alternativa econômica e ecologicamente viável que fornece um destino definitivo para esse lixo. Assim, nos últimos anos, vários estudos foram realizados visando a substituição parcial de materiais convencionais por resíduos de várias áreas. Em alguns casos, é possível desenvolver novos materiais e produtos com características físicas e mecânicas semelhantes e até maiores quando comparados aos materiais e produtos convencionais. A aplicação do vidro moído em pó como pozolano em materiais cimentícios começou nos anos 70, embora a maior parte do trabalho desenvolvido nesta área seja relativamente recente [1-5]. A adição de pó de vidro influencia a qualidade da mistura devido à melhor embalagem das partículas, favorecendo o preenchimento dos vazios entre os grãos. A atividade pozolânica é favorecida por partículas finas, menores que $75 \mathrm{~mm}$ [6].

\section{MATERIAIS E MÉTODOS}

O resíduo de vidro foi coletado na estação de tratamento de resíduos da empresa Viminas, localizada na cidade de Sierra, estado do Espírito Santo, Brasil. A fim de avaliar o efeito do uso de resíduos de vidro, várias argamassas foram formuladas e testadas. A composição e a porcentagem de adição de pó de vidro variaram, e foram comparadas com uma argamassa de referência. A Tabela 1 apresenta uma formulação das argamassas, é importante ressaltar que essas fórmulas seguiram uma relação ótima entre aditivo e cimento (a / c =0,7), que anteriormente foram especificadas em outros trabalhos [7].

Tabela 1. Formulação das argamassas estudadas e sua nomenclatura

\begin{tabular}{|c|c|c|c|c|}
\hline Nomenclatura & Cimento & $\begin{array}{c}\text { Residuo } \\
\text { (cimento) }\end{array}$ & $\begin{array}{c}\text { Areia } \\
\text { (areia) }\end{array}$ \\
\hline ACRef & 1,00 & 0,00 & 2,00 & 0,00 \\
\hline ACSC10 & 0,90 & 0,10 & 2,00 & 0,00 \\
\hline ACSC15 & 0,85 & 0,15 & 2,00 & 0,00 \\
\hline ACSC20 & 0,80 & 0,20 & 2,00 & 0,00 \\
\hline ACSA10 & 1,00 & 0,00 & 1,90 & 0,10 \\
\hline ACSA15 & 1,00 & 0,00 & 1,85 & 0,15 \\
\hline ACSA20 & 1,00 & 0,00 & 1,80 & 0,20 \\
\hline
\end{tabular}




\begin{tabular}{|c|c|c|c|c|}
\hline ACSC/A 10-10 & 0,90 & 0,10 & 1,90 & 0,10 \\
\hline ACSC/A 10-15 & 0,90 & 0,10 & 1,85 & 0,15 \\
\hline ACSC/A 10-20 & 0,90 & 0,10 & 1,80 & 0,20 \\
\hline ACSC/A 15-10 & 0,85 & 0,15 & 1,90 & 0,10 \\
\hline ACSC/A 15-15 & 0,85 & 0,15 & 1,85 & 0,15 \\
\hline ACSC/A 15-20 & 0,85 & 0,15 & 1,80 & 0,20 \\
\hline ACSC/A 20-10 & 0,80 & 0,20 & 1,90 & 0,10 \\
\hline ACSC/A 20-15 & 0,80 & 0,20 & 1,85 & 0,15 \\
\hline ACSC/A 20-20 & 0,80 & 0,20 & 1,80 & 0,20 \\
\hline
\end{tabular}

Além disso, as seguintes caracterizações foram realizadas para amostras de resíduos: a análise granulométrica foi realizada por técnicas de sedimentação e úmida. Análise mineralógica qualitativa por difração de raios $X$ (DRX) em um difratômetro modelo URD SEIFERT usando radiação Co em $40 \mathrm{KV}$ e $30 \mathrm{~mA}$ com ângulo de varredura $2 \theta$ de 6 a $60^{\circ}$. A análise química foi realizada por fluorescência de raios $X$ em um modelo Solar 969, equipamento Unicam. A atividade pozolânica foi obtida por testes de condutividade elétrica.

O teste de adesão das argamassas produzidas foi realizado de acordo com a Associação Brasileira de Normas Técnicas - ABNT [8-11]. As produções de argamassa também foram avaliadas sob diferentes condições de cura (Tabela 2), tendo sua adesão testada [12-13].

Tabela 2. Tempo e temperatura para os diferentes tipos de cura aplicados

\begin{tabular}{|c|c|c|}
\hline Tipo de Cura & Temperatura (으) & Tempo (dias) \\
\hline Normal & 24 & 20 \\
\hline Submersa & 25 & $14 / 14$ \\
\hline Aquecida & $24 / 70$ & \\
\hline
\end{tabular}

\section{RESULTADOS E DISCUSSÃO}

O ensaio de difração de raios $X$ do resíduo de vidro foi realizado para determinar as potencialidades e restrições para sua aplicação. A Figura 1 mostra o difratograma do resíduo e é possível verificar alguns minerais, ou grupo de minerais característicos: Sika (Quartzo), Caka (Calcário) e Alka (Alumina). Estes minerais não são considerados contaminantes e não prejudicam a aplicação do material como cimento ou agregado [14]. 


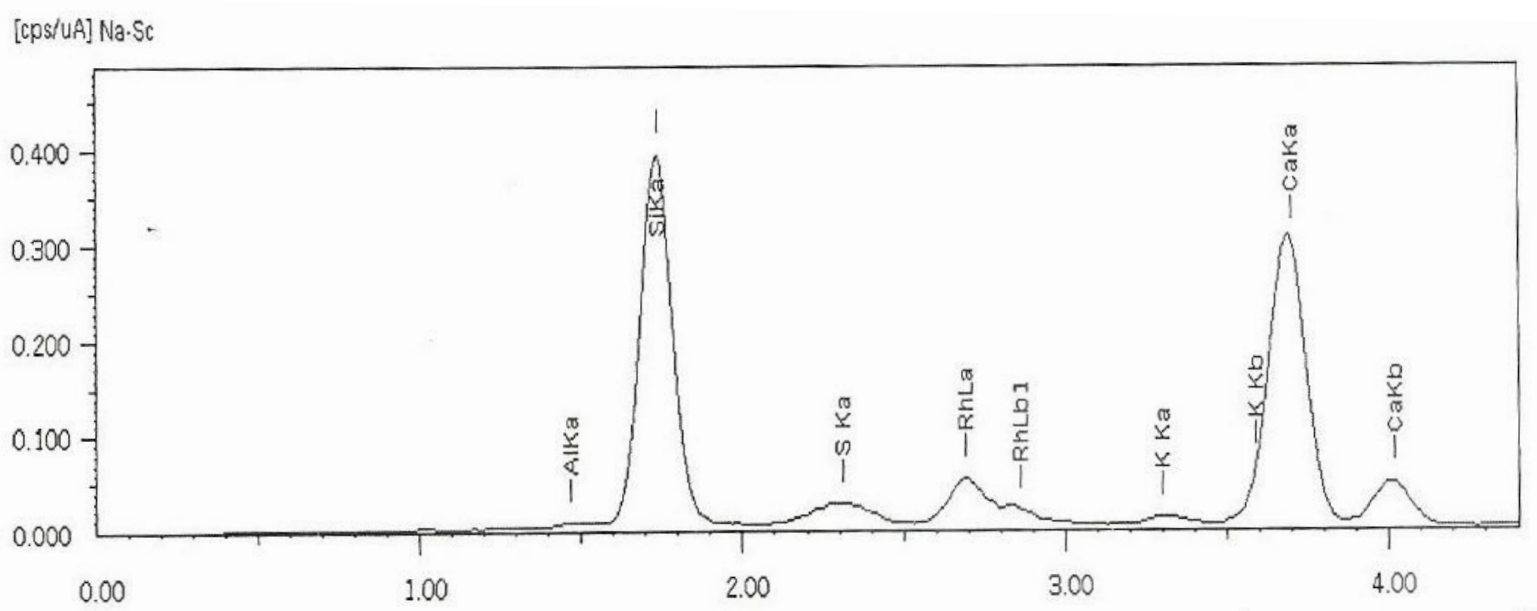

Figure 1. Difratograma do resíduo de vidro

A Figura 2 mostra a análise granulométrica do resíduo. Verificou-se que $70 \%$ das partículas estavam abaixo de $40 \mathrm{um}$, sendo $50 \%$ menor do que $10 \mu \mathrm{m}$. Conforme mencionado acima, isso irá favorecer a atividade pozolânica.

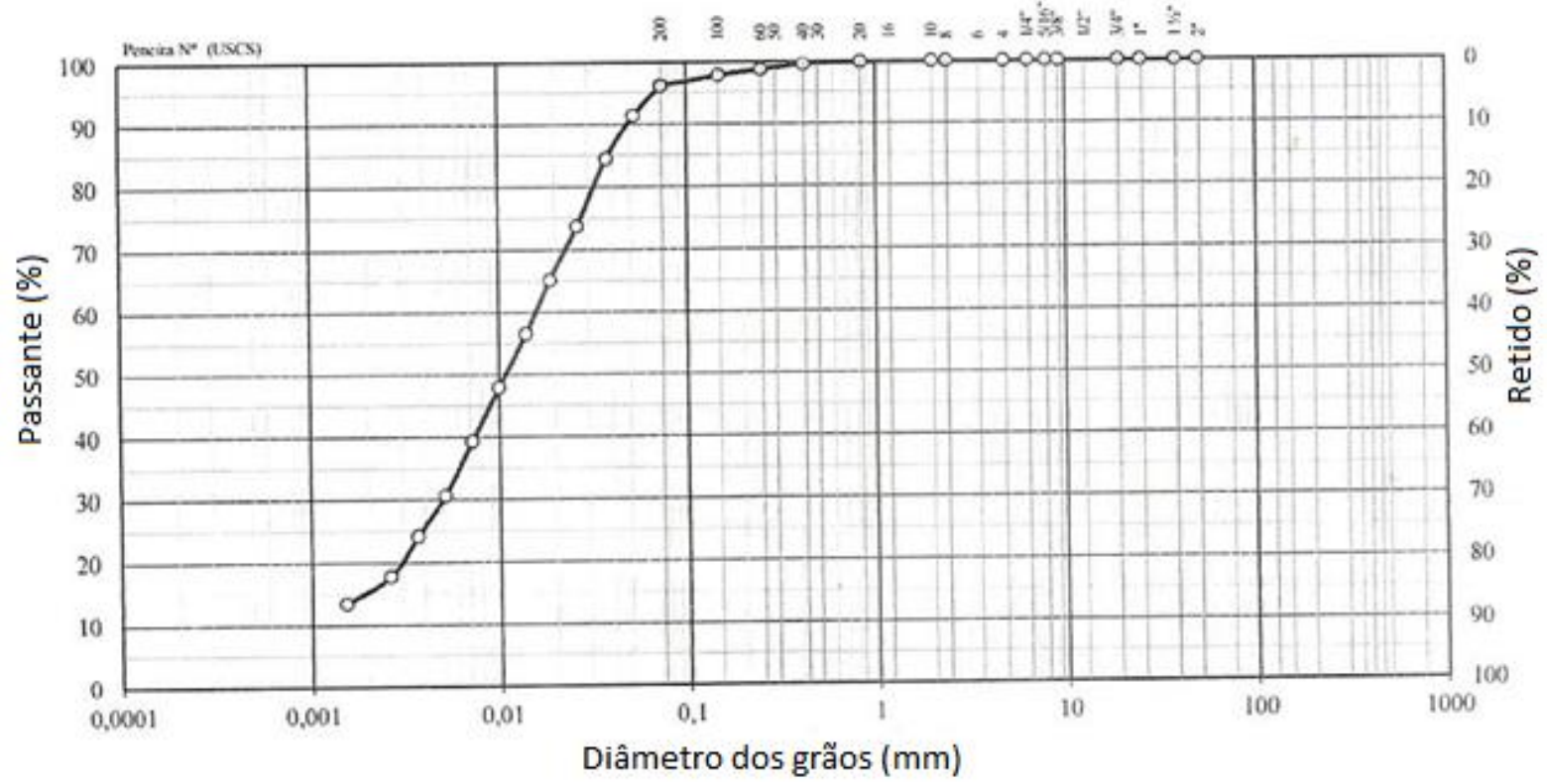

Figure 2. Distribuição do tamanho de partícula do resíduo de vidro

A análise química indicou que o resíduo de vidro consiste principalmente de $\mathrm{SiO} 2$ $(67,9 \%)$, Na2O $(13,6 \%)$ e $\mathrm{CaO}(8,4 \%)$, que correspondem a uma quantidade elevada de álcalis, típico do vidro comum de soda-lima.

A atividade pozolânica foi realizada por meio de testes de condutividade elétrica, como proposto por Luxan et al [15]. Os resultados apresentaram variação de condutividade na faixa de $1,24-1,56 \mathrm{mS} / \mathrm{cm}$, que na classificação de Luxan et al [15] representam uma boa atividade pozolânica.

A Figura 3 mostra os resultados médios da resistência à tração das argamassas. É possível observar que a argamassa com cimento e / ou areia substituída por resíduo de vidro atingiu resultados de resistência inferiores aos da argamassa de referência. No entanto, quase todas as argamassas podem ser classificadas positivamente de 
acordo com a norma brasileira [12] apresentando resultados superiores a 0,5 MPa. Tais resultados estão de acordo com os relatados por Penacho et al [16], que relaciona o aumento da porcentagem de resíduo de vidro com a redução da adesão da argamassa.

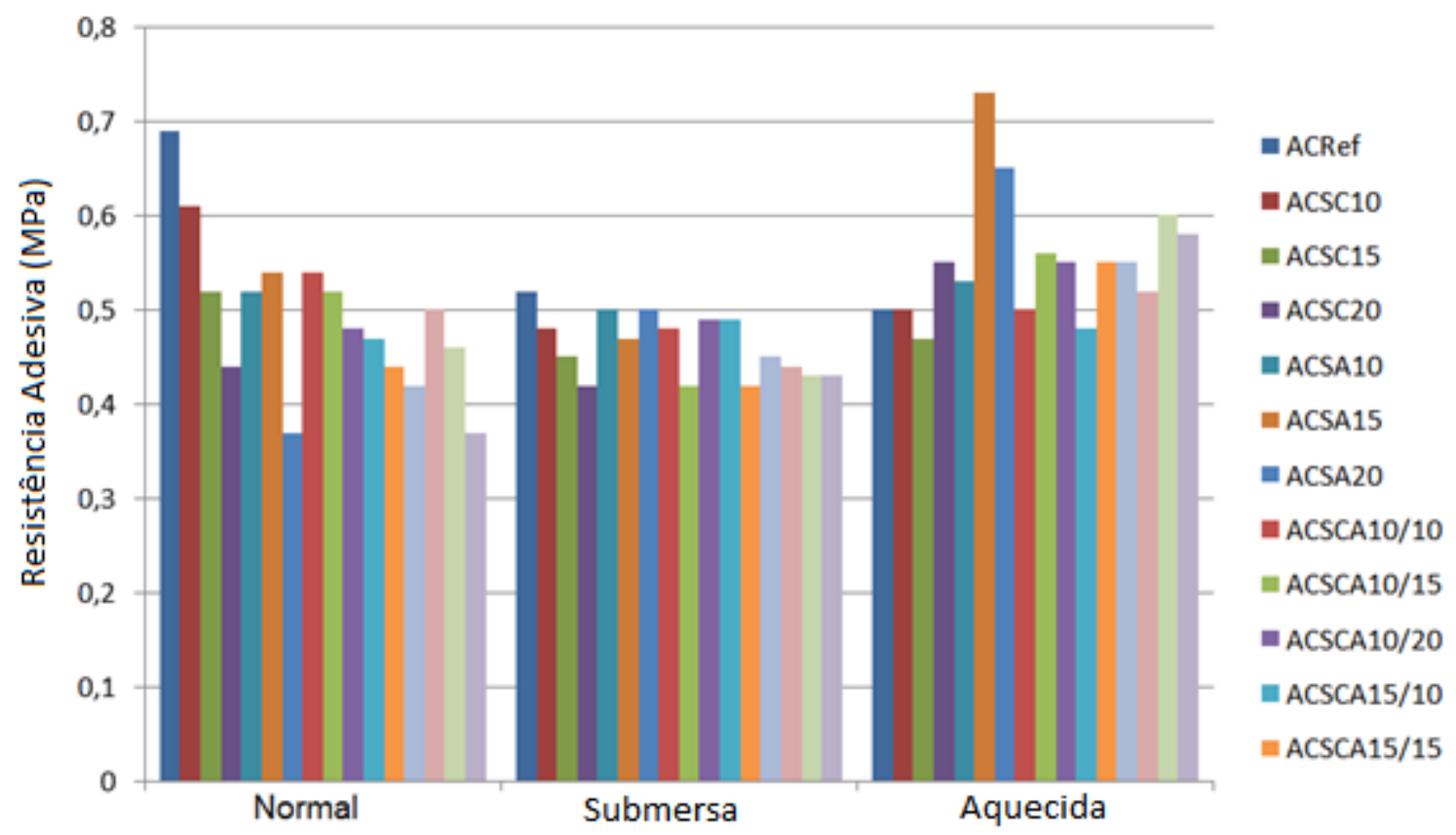

Figure 3. Resistência adesiva das argamassas obtidas de acordo com a norma ABNT

\section{CONCLUSÃO}

- O cimento e os agregados finos foram substituídos por resíduos de vidro na fabricação de argamassa. À medida que a quantidade de resíduo de vidro aumenta, verificou-se uma diminuição da consistência da argamassa quando comparada com uma argamassa de referência.

- A substituição conjunta de cimento e areia por resíduo de vidro resultou em uma resistência de adesão limitada das argamassas, mas os valores de resistência mínima relacionada ao padrão não foram atingidos.

- O aumento da temperatura de cura resultou em um ganho de resistência das argamassas em função do teor de substituição, uma vez que altas temperaturas favorecem as reações pozolanas devido ao resíduo de vidro.

- A diminuição da temperatura de cura resultou em valores mais baixos de resistência à adesão, que podem caracterizar uma vunerabilidade da argamassa em condições úmidas.

Com base neste trabalho, podemos concluir que é possível produzir argamassas de forma mais sustentável, através da substituição parcial de cimento ou areia por resíduos de vidro, sem perdas significativas de propriedades.

\section{Agradecimentos}

Os autores agradecem o apoio das agências brasileiras: CNPq, FAPERJ e CAPES para o desenvolvimento deste trabalho. 


\section{REFERÊNCIAS}

1 Shayan A, Xu A. Performace of glass powder as a pozzolanic material in concrete: A field trial on concrete slabs. Cement and Concrete Research. 2006; 36; 457-468.

2 Shi C, Zheng K. A review on the use of waste glasses in the production of cement and concrete. Resource Conserv Recycl. 2007; 52; 234-247.

3 Tan K. Use of waste glass as sand in mortar: Part I - Fresh, mechanical and durability properties. Cement Concr Compos. 2011.

4 Ling TC, Poon CS. Use of recycled CRT funnel glass as fine aggregate in dry-mixed concrete paving blocks. J Clean Prod. 2014; 68; 209-215.

5 Nassar RUD, Soroushian P. Strength and durability of recycled aggregate concrete containing milled glass as partial replacement for cement. Constr Build Mat. 2012; 29; 368-377.

6 Babu KG, Prakash PVS. Efficiency of silica fume on concrete. Cement and Concrete Research. 1995; 25; 1273-1283.

7 Santos DP, Azevedo ARG, Hespanhol RL, Alexandre J. Characterization of incorporation the glass waste in adhesive mortar. Characterization of Minerals, Metals, and Materials, John Wiley \& Sons Inc, USA. 2016; 539 - 545.

8 Associação Brasileira de Normas Técnicas. NBR 13276 - Argamassa para assentamento e revestimento de paredes e tetos - Preparo da mistura e determinação do índice de consistência. Rio de Janeiro, 2005.

9 Associação Brasileira de Normas Técnicas. NBR 14081-1 - Argamassa colante industrializada para assentamento de placas cerâmicas - Parte 1. Rio de Janeiro, 2012.

10 Associação Brasileira de Normas Técnicas. NBR 14081-2 - Argamassa colante industrializada para assentamento de placas cerâmicas - Parte 2. Rio de Janeiro, 2012.

11 Associação Brasileira de Normas Técnicas. NBR 14081-3 - Argamassa colante industrializada para assentamento de placas cerâmicas - Parte 3. Rio de Janeiro, 2012.

12 Associação Brasileira de Normas Técnicas. NBR 14081-4 - Argamassa colante industrializada para assentamento de placas cerâmicas - Parte 4. Rio de Janeiro, 2012.

13 Associação Brasileira de Normas Técnicas. NBR 14081-5 - Argamassa colante industrializada para assentamento de placas cerâmicas - Parte 5. Rio de Janeiro, 2012.

14 Mehta PK, Monteiro PJM. Concrete Microstructure, Properties and Materials. São Paulo, Brazil, 2008.

15 Luxán MP, Madruga F, Saavedra J. Rapid evaluation of pozzolanic activity of natural products by conductivity measurement. Cem Concr Res. 1989; 19; 63-68.

16 Penacho P, de Brito J, Rosário MV. Physico-mechanical and performance characterization of mortars incorporating fine glass waste aggregate. Cem Concr Compos. 2014; 50; 47-59.) 